\title{
Decision Processes for Trustworthy Software
}

\author{
Guenther Ruhe \\ University of Calgary
}

\begin{abstract}
A recent survey done across different engineering domains has revealed that support of decision-making is one of the key motivations for performing process modeling. Decisions are focal points at all stages of the software development lifecycle. The appropriateness of decisions made about methods, tools, techniques, activities and resources is of pivotal importance for the success (or failure) of a project. The discipline of software engineering decision support studies the processes of facilitating "good decisions". Besides effectiveness and efficiency, transparency is a key characteristic of the "goodness" of decisions.

This talk analyzes software engineering decision-making processes with emphasis on its transparency. From empirical studies it was shown that transparency of processes improves trustworthiness into solutions offered. This is especially true for solutions offered from decision support systems with comprehensive and complex computations done to generate candidate decision alternatives. More specifically, we discuss the transparency of decisions for the class of product release decision process. We provide the state-of-the art practice in this area and summarize existing research to address current deficits. Experience from a series of real-world projects in this area is analyzed from the perspective of how transparent processes have contributed to trustworthy software.
\end{abstract}

